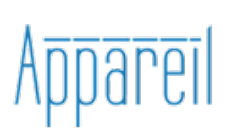

Appareil

$14 \mid 2014$

Esthétiques latino-américaines : penser à rebours

\title{
Sur une certaine fonction politique du cinéma latino-américain : L'étrange cas du regard poignant dans El compadre Mendoza de Fernando de Fuentes
}

Román Domínguez Jiménez

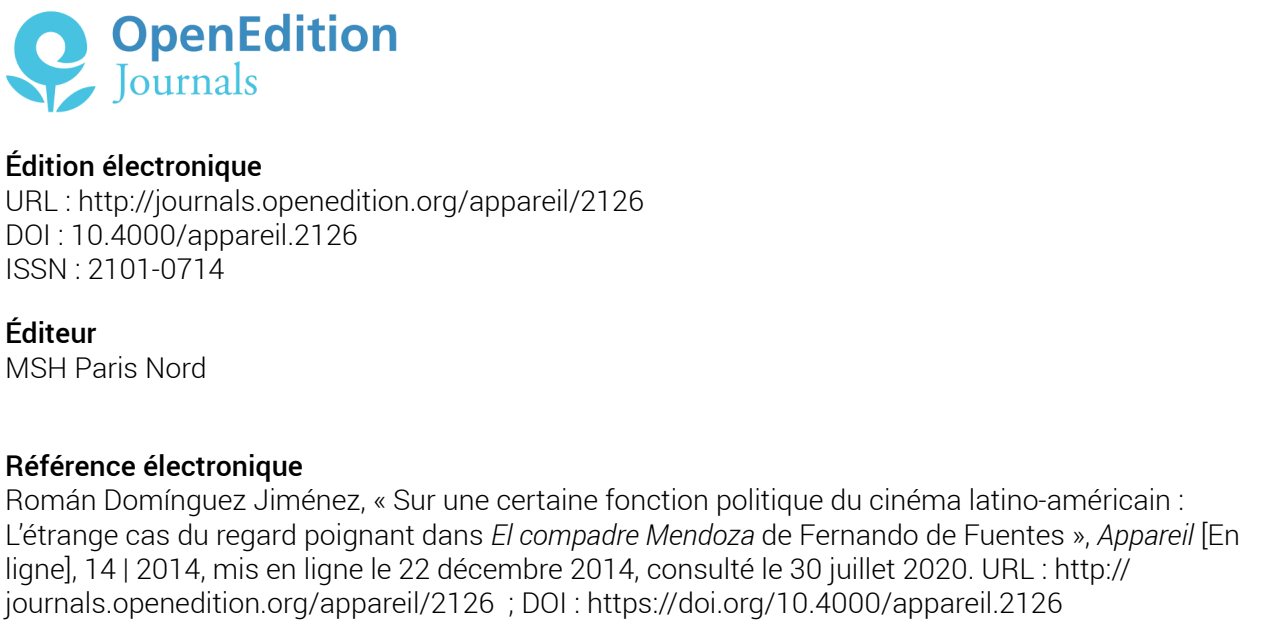

Ce document a été généré automatiquement le 30 juillet 2020.

\section{(i) $8=$}

Appareil est mis à disposition selon les termes de la Licence Creative Commons Attribution - Pas d'Utilisation Commerciale - Pas de Modification 4.0 International. 


\title{
Sur une certaine fonction politique du cinéma latino-américain : L'étrange cas du regard poignant dans El compadre Mendoza de Fernando de Fuentes
}

\author{
Román Domínguez Jiménez
}

\section{NOTE DE L'AUTEUR}

Ce texte est une version remaniée et adaptée au français (par mes propres soins) de mon intervention « Sobre una cierta función política en el cine latinoamericano: El extraño caso de la mirada en El compadre Mendoza de Fernando de Fuentes ", présentée dans le cadre du I Coloquio interdisciplinario de Estudios de Cine y Audiovisual Latinoamericano de Montevideo, Montevideo, Uruguay, les 11 et 12 septembre 2014, organisé par le groupe GEstA (Grupo de Estudios Audiovisuales) de l'Université de la République de l'Uruguay et Le Musée national des arts visuels de l'Uruguay.

1 Depuis quelques années, le cinéma et la mémoire politique semblent avoir scellé un pacte contre l'oubli, ainsi qu'une sorte de noce contre un présent hideux. Mais il se peut que de telles alliances ne soient que le résultat du développement, dans certains milieux intellectuels, d'une idée reçue, à savoir que certaines images auraient le pouvoir de nous émouvoir au point que, nous les spectateurs, deviendrions des sujets conscients des crimes, ou mieux, du crime qui, sous des masques diverses, se répète sans cesse dans l'histoire. Depuis Shoah de Lanzmann notamment, il est devenu presque évident de croire que cette conscience du crime, issue surtout du visionnage des témoignages, renvoie à une perception différente de celle du spectateur habituel des images de l'industrie culturelle. 
2 Notre texte veut déployer l'hypothèse suivante: la plupart des images cinématographiques, y compris celles qui sont censées être faites pour éviter l'oubli du crime, ainsi que pour empêcher que le pire de notre histoire recommence sous d'autres formes, ont tendance à ne devenir que des variations d'un maniérisme sentimental et mimétique qui s'est emparé de presque tout l'audiovisuel et des nouveaux medias comme les réseaux sociaux et des sites de partage des vidéos. Car au contraire du supposé assez répandu qui consiste à croire que nous manquons de mémoire, dans notre époque, le passé récent est devenu paradoxalement présent comme jamais dans l'histoire récente. Si bien qu'il est possible de dire que comme jamais auparavant, le passé exerce sur nous une hégémonie culturelle qui empêche la création d'une nouvelle politique. Il est vrai que cette hégémonie n'est pas celle de la mémoire historique, il s'agit plutôt d'un passé qui, dans la mesure où il s'est construit sur une base audiovisuelle, exerce son pouvoir d'une manière mimétique, purement sensuelle. Mais cette séduction tend à neutraliser toute singularité des images qui ont pour objectif de construire une mémoire politique. C'est qu'avec la diffusion croissante des nouveaux dispositifs et de la disponibilité à échelle universelle des enregistrements audiovisuels, nous avons commencé à développer une mémoire voluptueuse et mélancolique (tout à fait distincte d'une mémoire construite sur une base scripturale), dont la séduction nous pousse à consommer toute image, qui nous convie à expérimenter un film de témoignage presque comme s'il s'agissait d'un western. La conscience supposée du spectateur des films de témoignage ne serait dès lors qu'une variation pieuse du montage mélancolique de notre époque.

3 Le défaut probable de la plupart des films de la mémoire à cet égard serait d'avoir été construit sur la même base mimétique que le reste du cinéma : la sympathie envers l'un des côtés de l'histoire, celui des victimes. Mais aucune sympathie n'a été suffisante pour changer le cours des événements. Nous voulons proposer l'existence d'une autre fonction du cinéma : le point de vue critique. Ce point se déroulerait dans des passages où on ne l'attend pas, on le trouverait souvent dans une sorte de found footage. Pourtant, nous pensons que ce point de vue aurait la force d'établir un autre rapport entre notre présent et notre passé, afin de nous aider, non pas à faire mémoire, mais à faire face au crime. Nous croyons avoir trouvé l'un de ces points de vue dans un film qui concerne le devenir historique de l'Amérique latine : El compadre Mendoza (1933) de Fernando de Fuentes.

\section{Sur le sentimentalisme contemporain}

4 Nul doute qu'avec le défilement du temps, et notamment depuis l'avènement de la technologie numérique et de son relais par les réseaux informatiques, l'image en mouvement s'est assurée un statut privilégié en tant que document historique et en tant qu'interface archivistique. Nous pensons que ce statut-ci découlerait d'une certaine capacité ou fonction qui, bien qu'inhérente de longue date à l'image en mouvement, ne serait qu'aujourd'hui perceptible d'une manière plus limpide et nette. Il ne s'agit pas tellement de la fonction de témoignage que le cinéma partage d'ailleurs avec la photographie. C'est-à-dire, il ne s'agit pas ici du célèbre ça a été que Barthes accordait à la photographie dans La chambre claire ${ }^{1}$ (avant bien sûr que le numérique bouscule la capacité, partagée par la photographie et le cinéma analogiques, à rendre compte d'un réel plus ou moins cru), mais bien plutôt de cette autre faculté 
pseudomagique du cinéma, qui consiste, par le biais du montage, à ressusciter le passé : pour le meilleur et pour le pire, tantôt sur une tonalité séductrice, tantôt sur un mode atroce, que ce soit pour enfanter la nostalgie ou bien pour fabriquer un passé qui n'a jamais vraiment existé. Autrement dit: tandis que la temporalité traditionnelle de la photographie serait celle de l'évocation du passé (par exemple, dans un vieille photo des camarades du lycée), le plan cinématographique, ne possède pas d'ordinaire cette puissance évocatrice, car le mouvement cinématographique nous fait entrer dans un rapport au temps qui n'est pas tellement de l'ordre de la seule évocation, mais plutôt de l'ordre de faire vivre: vivre une chose, un événement (même s'il agit d'une illusion), c'est autre chose que l'évoquer. Dès lors, le passé du ça a été qui se présenterait d'une façon presque immédiate dans la photographie aurait peine à subsister au cinéma. C'est que l'évocation a besoin de la contemplation, d'un arrêt, d'une suspension des choses. Certes, il y a des passages et des procédés cinématographiques qui se prêtent mieux à l'évocation comme le ralenti. Mais nous croyons que la tendance générale du cinéma (dans la mesure où l'on peut dire que cette tendance est technique avant d'être culturelle ou artistique) est de marginaliser l'évocation comme forme du passé, au mieux de la laisser subsister dans quelques passages seulement. Cependant, le cinéma surmonte souvent ce handicap-là, par une voie qui ne se trouve pas directement dans le plan : par le raccord entre des plans, ou bien par d'autres opérations, comme certains faux raccords entre le visuel et le sonore. De telle sorte que le cinéma surpasse souvent d'emblée son défaut par le biais d'opérations de montage qui deviennent parfois des conventions techniques très répandues comme le flashback. Alors, il est possible de dire qu'au contraire de la photographie, et grâce à certaines opérations techniques, le cinéma peut éventuellement rendre présent, voire présentifier un passé. Cela peut être valable même pour les cas extrêmes des films mélancoliques et nostalgiques comme L'année dernière à Marienbad de Resnais - un film considéré par Deleuze comme l'exemple le plus achevé de l'image-cristal, c'est-à-dire, d'une image où passé et présent deviennent indiscernables ${ }^{2}$. En tout cas, cela vaut également pour des films d'Ingmar Bergman tels que Jeux d'été (Sommarlek) et Les fraises sauvages (Smultronstället). Le héros de chacun de ces films, autant la danseuse du premier film, que le vieux professeur du second, vivent à nouveau leur passé par le biais de flashbacks. C'est donc par cet aspect que le cinéma se différencie indéfectiblement de la photographie en ce qui concerne leur relation avec le passé : la photographie évoque un passé, dicte un cela a été : j'ai été à ce moment précis et dans cet endroit-là, avec mes amis et mes anciens camarades; tandis que le cinéma, notamment par le biais des conventions de montage comme le flashback, nous contraint à mener l'expérience du passé ; il nous invite à refaire ce qui a été, à revivre, répéter et même corriger ce qui a existé une fois (comme dans la machine du temps de Je t'aime, je t'aime, de Resnais).

5 L'une des conséquences politiques de ce statut (d'archive-document) et de cette fonction (présentifier le passé) a été l'institutionnalisation croissante du cinéma en tant que dispositif de mémoire historique, notamment en ce qui concerne le souvenir des guerres, dictatures et génocides. Mais il se trouve que les rapports entre l'image cinématographique et le temps ne sont jamais évidents. Il se peut que l'on ait construit cette institutionnalisation sur la supposition, en quelque sorte hâtive, d'un mécanisme d'empathie, à la fois trop général, voire grossier, et très insuffisant à l'égard d'un rapport politiquement productif et affirmatif entre l'image en mouvement et notre passé. Autrement dit, la fonction politique du cinéma concernant le passé, l'histoire, se trouve dans une grande mesure standardisée, formatée jusqu'à l'étouffement suivant 
un certain régime d'identification mimétique avec l'événement. Dans ce régime de standardisation mimétique, lui-même conditionné par l'opération technique de revivification du passé par l'image en mouvement, se faire une mémoire, se souvenir, serait équivalent à se situer dans un côté particulier de l'histoire, à soutenir les causes des victimes, à signaler et blâmer les bourreaux. Mais, si pertinentes que soient ces capacités qu'on a l'habitude d'accorder au cinéma, il se peut qu'aucune forme d'identification mimétique ne soit suffisante pour conjurer le trauma ou empêcher le retour du pire. Car l'humanité oublie; non pas tellement par manque d'une conscience morale supposée qui pourrait prétendument empêcher que le pire revienne encore une fois, mais plutôt parce que, comme Nietzsche l'a bien remarqué dans sa Deuxième considération inactuelle. De l'utilité et de l'inconvénient des études historiques pour la vie, tout renouvellement exige un certain oubli³. Dès lors le problème n'est pas l'oubli, qui dans une certaine mesure est nécessaire, et qui viendra forcément un jour, mais la configuration temporelle d'après laquelle nous oublions aujourd'hui. Configuration qui nous empêcherait d'entreprendre un travail de deuil authentique. Pour paraphraser un célèbre passage de Deleuze et Guattari dans Qu'est-ce que la philosophie ? ${ }^{4}$ : Nous ne manquons pas de mémoire, au contraire, due à la mise en forme du passé par des techniques audiovisuelles, nous en avons trop. Ce qui nous manque, c'est le travail de deuil et une critique politique du temps. Nous avons substitué une mémoire voluptueuse au désir d'une vie joyeuse à venir. Le travail de deuil devient donc impossible dans un régime sous lequel le rapport avec le passé s'institue non pas par la mémoire historique, mais par une mémoire mimético-sentimentale, c'est-à-dire par une mémoire tissée par une mélancolie plastique, audiovisuelle, voire par une mémoire rétro et vintage.

Précisons. Si on peut dire que cette démarche se trouve évidemment présente dans les films Agit-prop comme La hora de los Hornos des réalisateurs argentins Solanas y Getino, ou dans des documentaires comme $E l$ grito de Leobardo López Aretche sur la révolte étudiante et le massacre menée par l'État mexicain à Tlatelolco avant les Jeux olympiques à México en 1968, ou encore dans La batalla de Chile de Patricio Guzmán, les exemples plus clairs et frappants de ce que l'on peut appeler provisoirement la fonction mimético-politique de la mémoire dans le cinéma, se trouvent évidemment dans les films dont le sujet porte sur le génocide, des films qui depuis plusieurs années sont devenus des classiques du "cinéma de la mémoire »: S-21 La machine de mort Khmère rouge de Rithy Pahn sur le génocide au Cambodge, Calle Santa Fe de Carmen Castillo sur la dictature chilienne, et notamment les films de Claude Lanzmann (Shoah; Sobibor, 14 octobre 1943, 16 heures; Un vivant qui passe) sur la Shoah. Dans tous ces films, le montage ne se limite pas à construire un témoignage de faits insoutenables : il opère notamment un processus d'identification du spectateur aux victimes et corrélativement un sentiment de répulsion envers les bourreaux. Les séquences les plus célèbres de ces films sont les interviews par le documentariste des témoins et des victimes (Lanzmann) ou même des interviews des bourreaux (Lanzmann, mais surtout Rithy Pahn et récemment Joshua Oppenheimer dans The Act of Killing). Dans tous ces cas, on assiste à un Acting Out, à une performance parresiastique (Foucault), à une sorte de rictus par le biais duquel le témoin raconte une vérité, sa vérité, devant laquelle on ne peut pas rester indifférents.

7 Il ne s'agit pas ici de mépriser ni la fonction mimétique du cinéma en général (ce qui serait d'ailleurs impossible et inutile) ni son usage cathartique et limite, utilisé par exemple par Lanzmann lors de l'interview du combattant Yehuda Lerner dans Sobibor 
ou du coiffeur israélien Abraham Bomba (survivant d'Auschwitz) dans Shoah. Il s'agit d'avantage d'indiquer que malgré la fonction mimético-politique et malgré les louables efforts pour faire un cinéma politique (ou du moins un cinéma qui prétend apporter certains éléments pour une politique de la mémoire), cette manière de mettre en scène le passé s'égare dans la sensualité que l'image en mouvement suscite. Cela jusqu'au point d'avoir créé, au fil des années, un certain public "conscient», notamment universitaire, des situations limites : massacres, génocides, crimes d'État. Dès lors, il s'agit d'un public qui peut bien soutenir politiquement une cause contraire à celle des bourreaux, mais qui en même temps participe techniquement du même format d'identification du cinéma dit commercial. De telle sorte qu'il peut arriver qu'une image sur le témoignage et la mémoire politique et historique puisse être regardée, voire puisse être consumée presque de la même manière dont on regarde un western ou un autre film où les good guys luttent contre les bad guys.

\section{Envers le point de vue critique}

8 Ce que nous intéresse du point de vue d'une théorie critique et éventuellement par rapport aux réalisateurs eux-mêmes, c'est de développer les possibilités d'une politique $d u$ temps qui soit à la fois technique et esthétique. Une politique de l'image en mouvement qui cherche à établir un autre mode de fonctionnement du cinéma qui ne soit pas mimétique, ou du moins qui puisse préfigurer des autres facultés et possibilités mimétiques. Dès lors, l'objectif serait de trouver une spécificité du cinéma vis-à-vis des problèmes d'une politique de la mémoire qui puisse déplacer et relancer notre rapport politique avec le passé. Il nous semble qu'aucune sympathie (même la plus juste) n’a été suffisante pour arrêter le cercle pervers de la violence politique. Tout au contraire, tant qu'elle reste mimétique, la politique de la mémoire participera du système de la bonne conscience, lequel peut être employé pour des fins qui ne sont ni justes ni politiques, mais qui servent simplement l'industrie du divertissement, de la communication et de la culture. Il est vrai que ces industries sont immanentes à nos sociétés contemporaines, mais leurs fins ne peuvent pas remplacer la nécessité d'une autre politique à l'égard de nos manières de voir et de sentir. Ces fins ne peuvent pas non plus se substituer aux manières dont la justice politique doit se reformuler.

9 À ce sujet, il nous semble qu'il est possible de déceler l'émergence d'un autre type de fonction qui, même si elle passe par l'audiovisuel, ne serait plus miméticosentimentale, et constituerait davantage une mimesis déplacée, défigurée. Il s'agirait d'une fonction qui, au lieu d'exprimer une identification, constituerait, par le biais du jeu de caméras, des plans, et par le jeu de regards croisés notamment, un point de vue critique. Ici, il faudrait comprendre la notion de point de vue critique d'une manière littérale. C'est-à-dire ni comme une opinion sur un thème ou un sujet ni comme une fonction de discours qui renverrait à une théorie ou idéologie préétablie concernant la mémoire ou l'histoire (marxiste, par exemple), mais comme un point de vue optique et sonore qui distribue des signes au sein d'une temporalité critique, c'est-à-dire une temporalité instable, fragile, qui se déroule tout près du chaos, qui se compose donc comme une posture qui tient des signes face au chaos. La perspective de la Renaissance construit une temporalité, celle de l'instant (Déotte), à partir du rapport entre le point de vue (du spectateur) et le point de fuite (ou point du sujet) où les droites convergent et qui distribue les objets dans l'espace à partir d'une telle convergence ou projection. 
Mais le cinéma ajoute à la projection une dimension temporelle, parce que son point de vue est mobile (mouvement de caméra, mouvements des objets) et comprend aussi le découpage et le rapport entre le visuel et le sonore. Au contraire du point de vue de la perspective classique, le point de vue du cinéma est toujours une durée, un bloc d'espace-temps composé de mouvements et de changements des points de vue (par exemple dans l'opération de champ-contre-champ). De même, il s'agit d'un point de vue qui très souvent est imprégné par l'écoute (par exemple dans une voix off qui exerce une influence sur ce que l'on voit). On assisterait à l'émergence d'un point de vue critique lorsque les rapports mimétiques habituels (par exemple la reconnaissance d'un visage familier, du corps d'une victime, de l'attitude d'un bourreau) se trouvent suspendus au profit d'un rapport critique au temps, c'est-à-dire au profit d'un temps où le rapport entre des temps différents est en train de se faire, où le passé est encore vivant et où, dans cette mesure nous pouvons encore agir sur lui, non pas tellement sur les faits eux-mêmes, qui restent inchangeables, que sur le sens qu'ont pour nous ces faits, autrement dit, sur les faits en tant qu'événements dont le sens n'est pas encore scellé.

Tel que nous venons de l'exposer, le point de vue critique accomplirait une fonction de trompe-l'œil (et de trompe-écoute) par rapport à nos habitudes miméticocinématographiques, y compris par rapport à la fonction mimético-politique de la mémoire dans le cinéma. Car il ne s'agit plus d'une simple présentification du passé : par le biais des distances que compose le point de vue critique entre les plans, par les grandeurs qu'il manipule entre les éléments à l'intérieur du cadre et hors-cadre, par les rapports qu'il tisse entre les personnages (par exemple par un jeu de regards croisés ou par l'absence d'un tel jeu), le point de vue critique établirait un espace-temps non achevé, accompli et dès lors vivant, dont la fonction principale serait d'annoncer ou de nous prévenir d'un danger. Car il n'y aurait de moment ou de durée critique que par rapport à un danger, et il n'y a de danger vivant que là où le chaos, la mort, le pire menacent, que là où les choses, la vie, semblent s'effondrer. Le point de vue critique serait donc un fragment ou bloc de temporalité plastique qui nous avertirait d'une béance entre le passé et le présent sur laquelle il faut agir avec urgence, à l'instar des alarmes sismiques qui signalent un laps de temps (critique justement) pendant lequel la population peut se mettre à l'abri d'une catastrophe imminente, ou encore à l'instar des alarmes aériennes de la deuxième guerre mondiale, qui avertissaient la population d'un bombardement. Ainsi comprise, la fonction essentielle du point de vue critique ne serait pas de nous donner un rôle face à un destin historiquement déterminé, mais de nous mettre en état d'alerte lorsque ce point de vue met en suspens la destination des choses qui sembleraient fatalement certaines au cours de l'histoire elle-même. Certes, cette fonction ne serait pas tout à fait nouvelle dans le cinéma. On peut mentionner des cinéastes qui en ont fait usage assez récemment dans le cinéma de l'Amérique latine, comme Amat Escalante dans Heli (2013) à l'égard de la " guerre » contre les barons de la drogue au Mexique, ou encore Lucrecia Martel dans La Femme sans tête (La mujer sin cabeza, 2008), concernant la mémoire des disparus pendant la dernière dictature en Argentine. Surtout, on la retrouve d'une manière exemplaire et limpide dans une séquence du film mexicain de Fernando de Fuentes El compadre Mendoza (1934). 


\section{Le crime dans La mujer sin cabeza de Lucrecia Martel}

11 Examinons d'abord, en guise de préambule à l'analyse du film de Fernando de Fuentes, ce qui arrive dans le film de Martel: au début du film, dans la province argentine de Salta, une femme de classe moyenne, et qui semble avoir une quarantaine d'années, est au volant de sa voiture sur une route secondaire, conduisant au rythme d'un tube des années 1970. Elle écrase par mégarde ce qui pourrait être un enfant de classe populaire (elle voit par le rétroviseur ce qui pourrait être le corps de l'enfant, et nous avons vu auparavant que des gamins jouaient à cet endroit-là). Mais au lieu de s'arrêter, elle continue son chemin. Peu après elle s'arrête et sort de sa voiture pour se tranquilliser. Tout le long du film la femme sera hantée par le souvenir du crime qu'elle a probablement commis. Nous, les spectateurs, restons dans l'attente du moment crucial où l'on trouvera finalement le coupable et où peut-être justice sera faite. Au fur et mesure que le film se déroule, nous observons la protagoniste en plan rapproché, et souvent en gros plan: on la voit réfléchir, essayer de faire face aux situations qui pourraient la démasquer, craignant que son secret soit dévoilé. En même temps, on peut remarquer des signes divers qui nous rappellent l'existence de la victime supposée : des ombres et des silhouettes floues d'enfants qui passent et qui nous font penser à l'enfant probablement tué. Nous avons donc dans la plupart des cadrages un jeu de plans qui ne se touchent pas ou qui, au moment où ils entrent en rapport, nous déçoivent parce que ce nous attendons une toute autre chose: le rapport juste entre des plans qui renvoient à un rapport harmonique au niveau de notre pensée. La sagesse de Martel consiste déjà en empêcher la réconciliation entre le plan rapproché et l'arrière-plan, laissant déçues toutes nos attentes mimétiques de justice. Dès lors, le souvenir pénible imprègne ce qu'on voit et ce qu'on écoute. Tandis que la justice ne se voit pas, elle ne s'incarne pas, elle est invisible, elle habite nos pensées, qui se sont transformées dans le hors-champ absolu de ce qu'on voit.

Mais le plus important n'est sans doute pas là mais dans le corrélat de ce procédé : en empêchant la réconciliation, Martel développe un espace-temps où le spectateur est confronté à ses attentes concernant l'actualité et l'histoire de l'Amérique latine: la femme est blanche, criolla (descendante des migrants européens), dans une province où tous les subalternes ont la peau foncée (en tant que descendants des peuples originaires). Bien que la femme essaie parfois de faire face à la faute qu'elle vient de commettre, elle n'arrive pas à franchir le pas vers la vérité. Et lorsqu'elle avoue les faits à son mari, on ne trouve aucune trace du crime. Dès lors, ses proches l'aident à oublier, tandis qu'elle-même commence alors à croire, ou à feindre qu'elle croit, que rien ne s'est passé, que peut-être elle a écrasé un animal. C'est justement ce que l'on perçoit vers la fin du film: on commence à soupçonner que l'accident n'a pas eu lieu, que le corps tombé n'était pas forcément celui d'un être humain, qu'il aurait pu être celui d'un chien ou encore que la femme est paranoïaque et qu'elle a tout imaginé. Malgré tout cela, le spectateur sait bien que quelque chose est arrivé, et qu'au fil du temps les traces commencent à s'effacer, à se transformer en signes ambigus. Il est possible de dire que Martel ne se limite pas à construire une allégorie de la mémoire face aux disparitions de la dictature argentine, elle nous oblige davantage à expérimenter l'impuissance et même la lâcheté vis-à-vis de la disparition : comme si le crime demeurait toujours-là dans l'arrière-plan, en perturbant notre existence tout entière. Sans doute, c'est dans ce sens que nous sommes interpellés à agir : à partir de la réconciliation impossible entre les deux 
plans. Autrement dit, dans ce film Martel élabore deux lignes parallèles de plans qui ne se croisent qu'au moment de l'accident, et cela sur un mode non apparent sur le cadre, par le biais du montage : on écoute l'écrasement, on voit après ce qui pourrait être un corps. Les signes des deux plans ne se croisent pas d'une manière apparente, c'est le spectateur qui construit l'accident dans une image mentale (voir figure 1).

Figure 1. Schéma de rapports entre deux plans dans La mujer sin cabeza

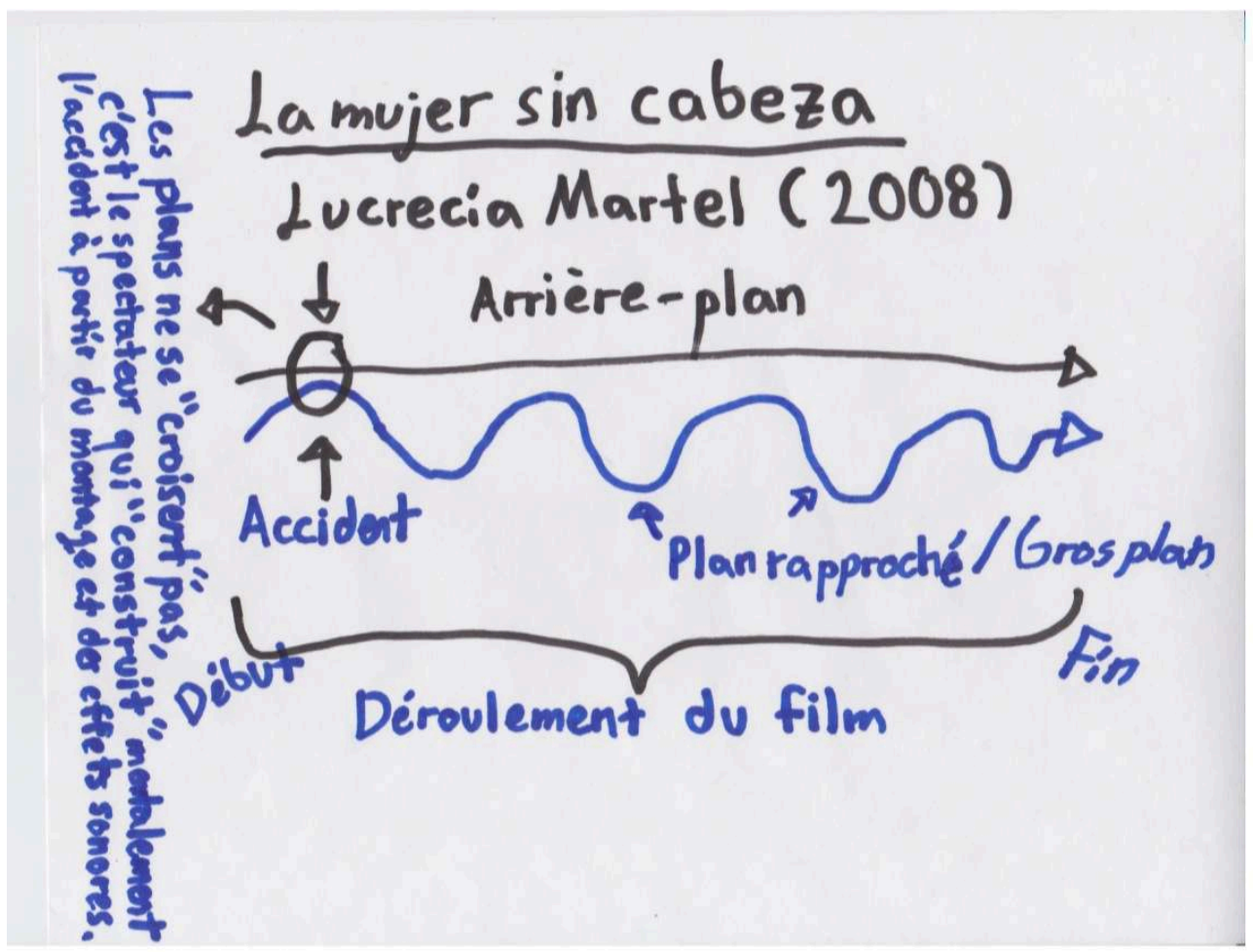

13 C'est cela l'expérience d'un point de vue temporel et critique à la fois : nous sommes éclaboussés dans notre pensée par un événement : l'audiovisuel ne nous donne pas une réponse, mais crée une tension, voire une suspension de nos jugements habituels. Il s'agit donc d'un point de vue critique, non seulement dans le sens où il exerce une critique théorique, mais surtout dans le sens où il se constitue comme un temps, une durée fragile et instable, à la fois rythmique et traumatique, qui permet au spectateur de voir et d'expérimenter des signes sociaux, politiques, historiques, discursifs en dehors de leur mécanique habituelle, comme si leur fonction normale avait été neutralisée. Point de vue ou espace-temps où la destination mimétique de l'image, de même que les destinations communicationnelles des signes se trouvent suspendues: nous n'expérimenterons pas une fois de plus la catharsis à laquelle nous sommes habitués; nous ne nous réconforterons pas dans notre soif de justice «de fauteuil»; nous ne découvrirons pas de signe qui puisse réparer la perte; nous ne trouverons aucune surface d'inscription pour les existences disparues. Alors, nos capacités de juger et de statuer sur une situation de façon définitive en tant que spectateurs se trouvent suspendues pour donner lieu à une critique de notre mécanique politique. C'est un peu comme si Martel nous invitait, non à simplement condamner le fait condamnable, mais à déconstruire de manière esthético-technique la mécanique qu'on mène quotidiennement : mécanique qui ne se définit pas par notre oubli, mais par notre faire semblant d'oublier, en ignorant ce qui a toujours été visible (les autres, le crime), même 
diffusément, parce que nous préférons les garder pour nous, à l'instar de nos petits secrets intimes, avec l'espoir que peut-être le temps arrivera à les effacer.

\section{Le regard de la servante dans El compadre Mendoza de Fernando de Fuentes}

Revenons maintenant à ce film ancien, El compadre Mendoza (1934), du réalisateur mexicain Fernando de Fuentes. Ce film nous intéresse à deux titres : d'abord parce qu'il montre une variation du point de vue critique différente et peut-être plus puissante que celle de Martel. Et ensuite parce qu'il montre qu'un tel point de vue peut être trouvé là où on ne l'attend pas. Car ce film n'a pas eu la prétention de nous interpeller, nous, les gens du xxI ${ }^{e}$ siècle, étant donné qu'il s'adresse aux spectateurs mexicains de l'époque postrévolutionnaire et cherche à leur montrer les échecs de la Révolution mexicaine (1910-1920). Fernando Fuentes est connu au Mexique pour ses films à caractère social, connus sous le nom de Trilogie de la Révolution : El prisionero trece (1933), El compadre Mendoza (1934) et ¡Vámonos con Pancho Villa! (1935), films qui montrent comment le déroulement et le destin de la Révolution passent par le malheur et la mort absurde de milliers d'anonymes. Mais très curieusement, de Fuentes est aussi l'un des pionniers du genre mélodramatique populaire mexicain : la Comedia Ranchera, laquelle, du moins en ce qui concerne l'histoire, n'est rien d'autre qu'une falsification, souvent caricaturale, de la campagne mexicaine. Il s'agit d'un genre où les rapports sociaux sont totalement mystifiés et absolument soumis à la domination de la figure du charro, le cow-boy mexicain, qui est par ailleurs l'archétype du macho mexicano, le mâle amoureux et courageux, mais aussi infidèle, bavard et dragueur. Et c'est justement le film célèbre de Fernando de Fuentes, Allá en el Rancho Grande (1936) qui inaugure la Comedia Ranchera aux traits de music-hall. Car l'une des spécificités du charro mexicain par rapport au cow-boy du western, c'est qu'il est toujours un chanteur accompagné des fameux mariachis. Pour résumer, on peut dire que s'il est vrai que de Fuentes n'est pas le meilleur réalisateur mexicain de l'histoire, il est néanmoins le plus important en ce qui concerne la généalogie du cinéma mexicain, justement parce qu'il est à la fois le grand cinéaste de la Révolution (jVámonos con Pancho Villa!) et son fossoyeur esthétique (Allá en el Rancho Grande).

Or, El compadre Mendoza porte sur une trahison qui renvoie à toutes ces autres trahisons qui devraient avoir eu lieu pour opérer la conversion assez singulière de la Révolution mexicaine en Parti révolutionnaire institutionnel (PRI). Il s'agit de l'histoire d'un hacendando (un fermier puissant et riche, propriétaire d'une hacienda ou rancho), Rosalío Mendoza, qui, afin de conserver son patrimoine et ses affaires, s'arrange toujours avec le pouvoir en place. Quand les forces du gouvernement fédéral prennent le pouvoir du territoire, Mendoza adhère à leur cause ; lorsque les rebelles zapatistes contrôlent les alentours, il change vite de position. De telle sorte qu'il devient ami et ensuite compadre (compère : un lien très important dans un pays majoritairement catholique comme le Mexique) du général zapatiste Felipe Nieto, juste pour éviter que les forces de l'armée rebelle l'exproprient. Mendoza signe son alliance en donnant à son fils le prénom du général : Felipe. Pendant les années de contrôle zapatiste dans la région, le général rebelle rendra visite assez souvent à Mendoza dans son ranch, tant par véritable amitié que pour avoir l'occasion de rencontrer sa comadre (commère), la femme de Mendoza, dont il est tombé amoureux : elle est jeune et belle, au contraire de son mari, vieux et 
décrépi. Mais la fortune des zapatistes dans la région changera bientôt. Un jour, un général des forces du gouvernement fédéral, rend visite à Mendoza et lui propose de trahir son compère : si Mendoza accepte, il pourra conserver son patrimoine, s'il refuse, l'armée mettra la main sur une partie de son négoce. Dans un premier moment, Mendoza s'indigne de subir un tel chantage, mais après avoir un peu réfléchi, il accepte la proposition et prépare une embuscade : il invite chez lui le général zapatiste pour une réunion avec le général de l'armée fédérale. Le zapatiste viendra tout seul, l'armée fédérale l'arrêtera et le condamnera à la pendaison. La nuit de l'embuscade sera orageuse, pleine d'éclairs et de tonnerre. Un peu avant de consumer la trahison, Mendoza se tient dans la pièce voisine à celle où l'attendent les deux généraux. Il demande alors à son valet d'aller se coucher, puis, il ordonne à sa femme et son fils de partir du ranch. Il reste dans l'habitation pour se donner du courage, mais un personnage l'accompagne encore : il s'agit de sa servante muette, laquelle soupçonne le pacte hideux de Mendoza. Celui-ci feint de ne pas la voir, après tout, il ne s'agit que d'une servante: femme, pauvre, ignorante et en plus muette. Il marche d'un côté à l'autre de la pièce, mais il commence à se sentir regardé. Il se tourne pour la regarder puis continue tout de suite à marcher d'un côté à l'autre. Il s'arrête et regarde la servante encore une fois. Elle sait bien ce que Mendoza va accomplir et jette sur lui l'un des regards les plus puissants de toute l'histoire du cinéma: pendant quelques secondes, on verra le visage de la servante en gros plan, on entendra en même temps le tonnerre, le plan changera pour nous montrer un très gros plan des yeux de la femme. Évidemment, elle ne dit rien, mais nous ressentons inévitablement dans son regard muet et presque inexpressif toute la force de la condamnation morale envers la bassesse de Mendoza. Celui-ci désespère, il commence à crier : "Qu'est-ce que t'as à me regarder? » (¿Qué me miras ?), " fous le camp dans ta cuisine ! » dit-il. La femme obéit et sort lentement, non sans continuer à regarder le traitre. Mendoza entre dans la chambre où l'attend son compadre et l'autre général et consomme peu après la perfidie.

Séquence :

Ce média ne peut être affiché ici. Veuillez vous reporter à l'édition en ligne http:// journals.openedition.org/appareil/2126

Résumons brièvement cette séquence : il est clair que le rôle de la servante est bien secondaire par rapport à la trame du film, mais en termes affectifs et critiques (au sens où nous venons de le développer), nous suggérons que le sens profond du film tourne et se déploie autour de la force centrifuge de ce passage-ci, car à ce moment précis la destination du récit et les signes mimétiques qui l'accompagnent se trouvent suspendus : un peu comme si l'histoire du Mexique s'arrêtait pendant un laps de temps très bref pour nous laisser l'entrevoir de plus près et au ralenti (voir figure 2). 
Figure 2. Schéma du développement gestuel par rapport à la scène du regard de la servante dans $E I$ compadre Mendoza

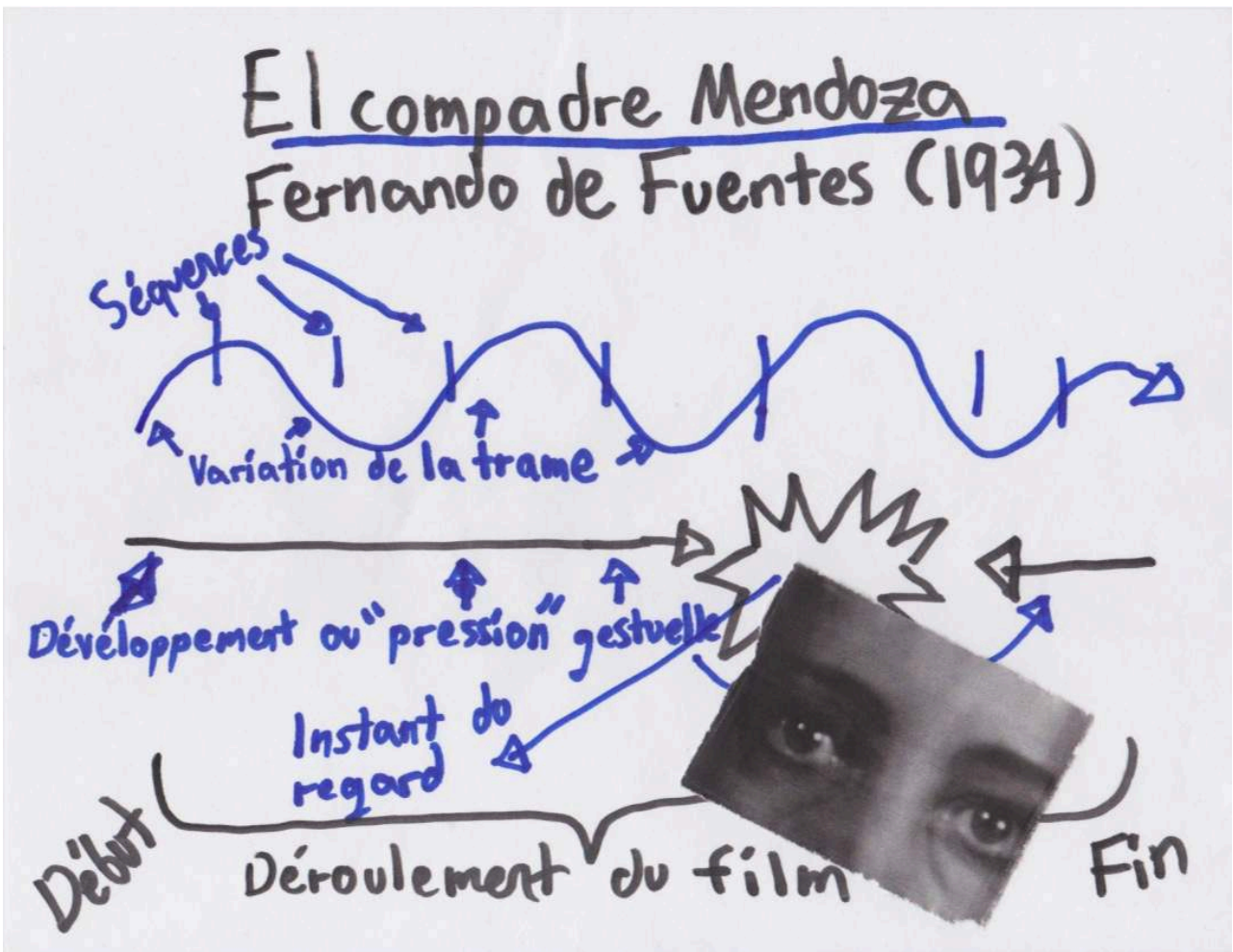

Assurément, même sans ce moment marquant où la servante fixe et accable moralement son maître par la force de son regard, El compadre Mendoza serait encore un film mémorable pour plusieurs raisons, mais il n'aurait probablement pas la force de nous convoquer à un rendez-vous inopiné avec notre passé. Ce passé concerne notamment le Mexique, un pays où les dettes historiques non résolues par la Révolution reviennent aujourd'hui sous une forme épouvantable avec une guerre qui a déjà provoqué des dizaines de milliers de morts. Et sans doute ce passé concerne-t-il aussi l'histoire de l'Amérique latine dans son ensemble, dans la mesure où elle s'est constituée, tissée au fil de grandes et petites trahisons comme celle du compadre Mendoza (Bolivar, O’Higgins, Allende, Arbenz du côté des trahis; Santa Anna et Pinochet du côté des traîtres). Témoin muet de la trahison historique, l'instant du regard de la servante constitue donc aujourd'hui, et pour nous, tout le contraire d'une catharsis mimétique du spectateur envers un des personnages. Et aussi tout le contraire d'une opération qui vise à fixer une fois pour toutes le sens du passé. Il est vrai que l'ensemble de la trame du film peut s'avérer traditionnellement mimétique (il est difficile, même pour le public de nos jours, de sympathiser avec un traître), mais ce n'est pas le cas du regard de la servante, puisque contrairement à ce qui arrive d'habitude avec la fonction mimético-politique, nous ne pouvons ni prendre la place de son regard ni prendre la place de celui qu'elle regarde (cette place restant réservée au bourreau ou au traître); c'est plutôt par la médiation de la force de ses yeux en très gros plan que nous nous sentons obligés de nous regarder nous-mêmes et notre histoire. 


\section{Notes pour le rendez-vous avec un passé intempestif} exprimer une position différente et un autre type de rapport à l'égard du crime : au lieu de forger une mémoire, c'est-à-dire, de tomber dans la prétention de construire des images censées nous aider à éviter que les crimes de l'histoire se répètent sur d'autres formes, voire que le pire de l'histoire recommence, il s'agit, avec la fonction du point de vue critique, de faire face au crime par un regard qui, d'une manière allégorique, intègre l'histoire et le destin de l'Amérique latine. De sorte que la séquence de El compadre Mendoza, où le regard de la servante affronte le maitre-traitre, ne nous apporte pas une rédemption de la violence atavique ou contemporaine, et ne rend pas davantage justice aux victimes. Elle nous donne d'avantage la possibilité d'un temps audiovisuel critique capable de faire face au trauma, à la blessure historique, pour accomplir un indispensable travail de deuil. Nous pensons qu'une politique digne de ce nom doit créer quelque chose de nouveau, mais pour créer il faut oublier, mais avant d'oublier il faut avoir rendu justice au passé par une action dans le présent, par une action qui puisse éventuellement guérir (du moins de manière partielle) le trauma.

Finalement, nous suggérons que les yeux de la servante ne jugent pas. Au contraire, ils mettraient en suspens le jugement du mimétisme, pour nous donner l'occasion d'élaborer un travail de deuil. Ce travail-ci ne devrait pas toutefois se produire sur un mode mortuaire, solennel et au profit des seules institutions culturelles, mais comme 
tâche indispensable, autant pour le redressement urgent du sens de l'histoire, que pour la recherche d'une rédemption qui puisse être réalisée avec les moyens techniques et politiques dont l'humanité dispose aujourd'hui. Benjamin souligne dans sa seconde thèse sur l'histoire, qu' « il existe une entente tacite entre les générations passées et la nôtre. Sur Terre nous avons été attendus $»^{6}$. Notre destin (et celui de nos enfants) dépend peut-être de ce rendez-vous mystérieux. Mais pour y arriver à temps, il faudra d'abord relancer notre rapport avec le passé et le temps en dehors de toute réconciliation mimétique et qui sait, sans mélancolie peut-être. Il se peut que notre destin et celui de nos ancêtres n'aient pas encore été scellés. Pour cela, nous pensons qu'il nous a été donné la force de décider selon quelle figure du temps nous voulons nous regarder nous-mêmes : celle du circuit éternel et violent du même dans l'histoire, ou celle de la ligne dévastatrice et intempestive d'une éventuelle libération à venir, où sans doute une paire de beaux yeux nous attend.

\section{BIBLIOGRAPHIE}

Barthes Roland, La chambre claire. Notes sur la photographie, Paris, Cahiers du cinéma / Gallimard, 1980.

Benjamin Walter, Gesammelte Schriften III, édition de Rolf Tiedemann et Hermann Schweppenhäuser, Francfort-sur-le-Main, Suhrkamp, 1991.

Benjamin Walter, CEuvres III, trad. de l'allemand par Maurice de Gandillac, Rainer Rochtlitz et Pierre Rusch, Paris, Gallimard, coll. « Folio Essais », 2000.

Deleuze Gilles Cinéma 1. L'Image-mouvement, Paris, Minuit, 1983.

Deleuze Gilles, Cinéma 2. L'Image-temps, Paris, Minuit, 1985.

Deleuze Gilles et Guattari Félix, Qu'est-ce que la philosophie ? Paris, Minuit, 1991.

Nietzsche Friedrich, Deuxième considération inactuelle. De l'utilité et de l'inconvénient des études historiques pour la vie, traduction Henri Albert, Paris, éditions Garnier-Flammarion, 1998.

Films cités :

Bergman Ingmar, Jeux d'été (Sommarlek), Suède, 1951.

Bergman Ingmar, Les fraises sauvages (Smultronstället), Suède, 1957.

Castillo Carmen, Rue Santa Fe (Calle Santa Fe), Chili, France, Belgique, 2007.

de Fuentes Fernando, Le prisonnier treize (El prisionero trece), Mexique, 1933.

de Fuentes Fernando, El compadre Mendoza, Mexique, 1934.

de Fuentes Fernando, ¡Vámonos con Pancho Villa!, Mexique, 1935.

de Fuentes Fernando, Allá en el Rancho Grande (première version), Mexique, 1936.

Escalante Amat, Heli, Mexique, Pays-Bas, Allemagne, France, 2013. 
Guzmán Patricio, La bataille du Chili (La batalla de Chile), Venezuela, France, Cuba, 1975, 1976, 1979

(film sorti en trois parties).

Lanzmann Claude, Shoah, France, 1985.

Lanzmann Claude, Un vivant qui passe, France, 1997.

Lanzmann Claude, Sobibór, 14 octobre 1943, 16 heures, France, 2001.

López Aretche Leobardo, Le cri (El grito), Mexique, 1968.

Martel Lucrecia, La femme sans tête (La mujer sin cabeza), Argentine, France, Italie, Espagne, 2008.

Oppenheimer Joshua, The Act of Killing, Danemark, Norvège, Royaume-Uni, 2012.

Pahn Rithy, S-21, la machine de mort Khmère rouge, France, Cambodge, 2003.

Resnais Alain, L'année dernière à Marienbad, France, Italie, Allemagne Fédérale, Autriche, 1961.

Resnais Alain, Je t'aime, je t'aime, France, 1968.

Solanas Fernando Ezequiel et Getino Octavio, L'Heure des brasiers (La hora de los hornos), Argentine, 1968.

\section{NOTES}

1. Roland Barthes, La chambre claire. Notes sur la photographie, Paris, Cahiers du cinéma / Gallimard, 1980.

2. Gilles Deleuze, Cinéma 2. L'Image-temps, Paris, Minuit, 1985, chapitre IV «Les cristaux de temps », p. 92-128.

3. Friedrich Nietzsche, Deuxième considération inactuelle. De l'utilité et de l'inconvénient des études historiques pour la vie, traduction Henri Albert, Paris, Éditions Garnier-Flammarion, 1998.

4. « Nous ne manquons pas de communication, au contraire nous en avons trop, nous manquons de création. Nous manquons de résistance au présent ». Gilles Deleuze et Félix Guattari, Qu'est-ce que la philosophie? Paris, Minuit, 1991, p. 104.

5. Gilles Deleuze, Cinéma 1. L'Image-mouvement, Paris, Minuit, 1983, chapitre VI «L'imageaffection : visage et gros plan », p. 125-144.

6. Walter Benjamin, « Über den Begriff der Geschichte », in Gesammelte Schriften III, Francfort-surle-Main, Suhrkamp, p.694: «dann besteht eine geheime Verabredung zwischen den gewesenen Geschlechtern und unserem. Dann sind wir auf der Erde erwartet worden ». Version française : "Sur le concept d'histoire ", Deuxième thèse, in Euvres III, trad. de l'allemand par Maurice de Gandillac, Rainer Rochtlitz et Pierre Rusch, Paris, Gallimard Folio, p. 428.

\section{RÉSUMÉS}

Dans quelle mesure le cinéma peut-il contribuer à la lutte contre l'oubli voire faire en sorte que le pire de l'histoire ne se répète plus jamais ? Il convient d'examiner l'idée reçue selon laquelle il suffirait que le spectateur compatissant voie les crimes et entende les témoignages pour que l'horreur ne se reproduise plus. À partir d'une analyse du film El compadre Mendoza de F. de 
Fuentes, l'auteur critique le présupposé mimétique et développe un point de vue qu'il appelle critique. Ici, il ne s'agira plus de faire mémoire pour que le pire ne revienne plus, mais de faire face au crime par le biais des images (du passé notamment) qui nous mettraient en garde contre les dangers de nos jours.

INDEX

Mots-clés : politique, cinéma latino-américain, crime, histoire, mimesis

\section{AUTEUR}

ROMÁN DOMÍNGUEZ JIMÉNEZ

Maître de conférences (profesor asistente) à l'Institut d'Esthétique de la Pontificia Universidad Católica de Chile, rdominguezj@uc.cl 